\title{
Making infrared photometry accessible
}

\author{
M. S. Bessell \\ Mount Stromlo and Siding Spring Observatories, ACT 2611, Weston Creek Post Office, Australia \\ e-mail: bessell@mso . anu . edu . au
}

In 1966, Harold Johnson published a groundbreaking paper on the infrared properties of stars in Annual Reviews of Astronomy and Astrophysics. It brought together broadband photometry from $U$ to $N(0.36$ to $10.2 \mu)$. Its emphasis was on the infrared part of the spectrum that at that time comprised the near-IR (visible to $1 \mathrm{~m}: V, R$, and $I$ bands), intermediate-IR (1-4 m: $J K L$ bands) and the far-IR (4-22 m: $M$ and $N$ bands). These are now called the far-red, near-IR, and IR, respectively. This was based on observations of mainly bright stars using an S1 phototube for $R$ and $I$, a PbS cell for 1-4 $\mathrm{m}$, and a germanium bolometer for 4-22 m observations.

This highly cited paper stimulated great interest in the possibilities of red and IR observations of stars and galaxies, although technical difficulties of instrumentation and the high noise of the detectors restricted IR observations at the time to large telescopes and/or bright stars. But in the mid 1970s the advent of the InSb photovoltaic diode cooled with liquid nitrogen enabled faint stars to be observed between 1 and $4 \mathrm{~m}$ with excellent precision, and the era of modern infrared astronomy took off. Now, of course, the InSb diode, like the photomultiplier tube, has been superceded by arrays such as the various low-noise $\mathrm{HgCdTe}$ devices.

By $1980-82$, precise standards in the $J(1.25 \mu), H(1.65 \mu)$, $K(2.2 \mu), L(3.6 \mu)$, and $M(4.8 \mu)$ (fewer in $M$ where the high thermal telescope background greatly limits observations) had been established by several observers at different observatories. However, unlike optical photometrists, IR astronomers maintained their natural photometric systems and did not transform their photometry into a single standard system other than attempt to get close to the original Johnson $J K L M$ zero-point for an A0 star. As a result, IR photometry and standard magnitudes were published in different, albeit similar, systems making inter-comparisons difficult, confusing to the uninitiated, and unnecessarily imprecise.

Jan Koornneef was the first astronomer to address this issue. In Paper I in A\&AS 51, 489 he provided a homogenized list of southern stars with JHKLM photometry, and in his highly cited Paper II in A\&A 128, 84, used that data to update the very useful Johnson tables of intrinsic IR colors for different spectral types. Koorneef also provided a revised zero-point flux calibration for zero magnitude in the homogenized $J, H, K, L$, and $M$ bands and modified the useful relations devised by Allen (1981) to predict the absolute spectrophotometric flux of early $\mathrm{G}$ dwarfs in the $J, H, K$, and $L$ windows from the measured $K$ magnitude. These relations continue to be invaluable for providing flux calibration for IR spectrophotometry.

There are interesting discussions in the paper comparing the observed IR colors of stars with the computed colors of blackbodies and the "new" Kurucz (1979) ATLAS model atmosphere fluxes. The nature and problem of interstellar reddening in the near IR $(1-4 \mu)$ was also discussed and an adopted near-infrared reddening law presented. Koorneef noted that precise synthetic magnitudes and color-color relations required the proper convolution of passbands with stellar and model fluxes rather than deriving monochromatic fluxes at the nominal effective wavelengths of the JHKLM passbands that he had done; however, he was able to provide deep insight into the IR colors of stars with his analysis.

The rapid increase in the quality and quantity of IR observations over the next few years would enable the Koornneef analysis of IR photometry to be reappraised and extended. Glass (1985) did this, but as it was published in a much less visible journal than A\&A, it attracted little attention at the time. Bessell \& Brett (1988) finally provided passbands for the IR systems and convolved them with fluxes to determine more precise synthetic photometry as first noted by Koornneef.

Koorneef's 1983 A\&A paper was a very timely contribution that overcame the parochialism and insularity of many IR astronomers and made IR photometry accessible and quantitative to non-experts for the first time.

\section{References}

Allen, D. A. 1981, AAO IRPS User's Guide

Bessell, M. S., \& Brett, J. R. 1988, PASP, 100, 1134

Glass, I. S. 1985, Irish AJ, 17, 1

Johnson, H. R. 1966, ARA\&A, 4, 193

Koornneef, J. 1983, A\&AS, 51, 489

Koornneef, J. 1983, A\&A, 128, 84

Kurucz, R. 1979, ApJS, 40, 1 